\title{
Monitoring System for Prisoner with GPS using Wireless Sensor Network (WSN)
}

\author{
Pooja B. Patil \\ LATE G. N. Sapkal \\ College of \\ Engineering
}

\author{
Sneha Chapalkar \\ LATE G. N. Sapkal \\ College of \\ Engineering
}

\author{
Neha D. Dhamne \\ LATE G. N. Sapkal \\ College of \\ Engineering
}

\author{
Niharika M. Patel \\ LATE G. N. Sapkal \\ College of \\ Engineering
}

\begin{abstract}
In day today's life security is a very important thing in the world. For better achievement of security advanced technology have been undertaken in correctional facilities. Various security devices like CCTV, Tracking bracelets, ID barcode scanning and other devices have contributed to the improved and secure management.

The aim of this paper is to invent a security system against prisoner by using RF Transmitter and receiver technology. It is based on RF Transmitter and Receiver technology on ultra high frequency range which can be applied to use in access control by using RF Transmitter and Receiver tag. The RF Transmitter and Receiver tag contains a unique set of number as a code, so it can be identified. The RF Transmitter will act as the bracelet and the RF Receiver will be at the server side. For keeping the track of prisoner Global Positioning System (GPS) is used.
\end{abstract}

This technology can be used for monitoring the prisoners in the jail, patients in the hospitals or mental asylums who basically try to escape or run away from jails and mental asylums. It tracks the particular person wearing the bracelet within its range and as soon as it's outside the range the GPS connected to the bracelet gets activated and we can track that particular person with the help of GPS on the bracelet. It's also very useful in the schools for students and the staff, colleges, employees in the offices and etc where we need to keep track of someone's presence. There is no need of keeping continuous watch due to this technology, for example if the prisoner runs away from the jail a message will be sent to the jailer and buzzer will ring. Due to this technique it will be easy for monitoring purpose.

\section{Keywords}

RF Transmitter, RF Receiver, Bracelet, Security, Tracking.

\section{INTRODUCTION}

The topic is basically implementing perception for security purpose which is now a days has become a major issue .The topic describes security against theft. There are many prisoners who are in jail but there are some prisoners who somehow manage to escape from jail, which after running from jail continues their crime. The topic security bracelet is helpful to keep the track of the prisoner; bracelet will be tied on prisoner's wrist which will keep track of the prisoner and if prisoner tries to escape will detect it and will send message to jailer that a prisoner ran away, even buzzer is provided in the system. For implementing this system RF transmitter and receivers, GPS are used.

\subsection{RF Transmitter and Receiver}

The RF (Radio Frequency) module, as the name suggests, operates at Radio Frequency which describe the use of wireless communication. The RF has a rate of oscillation in the range of around $3 \mathrm{kHz}$ to $300 \mathrm{GHz}$ that corresponds to the alternating current which carry radio signals and to the frequency of radio waves. In this Radio Frequency system, the digital data is represented as variations in the amplitude of carrier wave. Such a kind of modulation is known as Amplitude Shift Keying (ASK).Transmission through RF is better than IR (infrared) because of many reasons such as; to begin with, signals through RF can travel through larger distances making it suitable for long range applications. Also, $\mathrm{RF}$ signals can travel even when there is an obstruction between transmitter \& receiver, while IR mostly operates in line-of-sight mode. Next, RF transmission is more reliable and strong than IR transmission. RF communication uses a particular frequency unlike IR signals which are affected by other IR emitting sources.

\section{Pin Diagram}

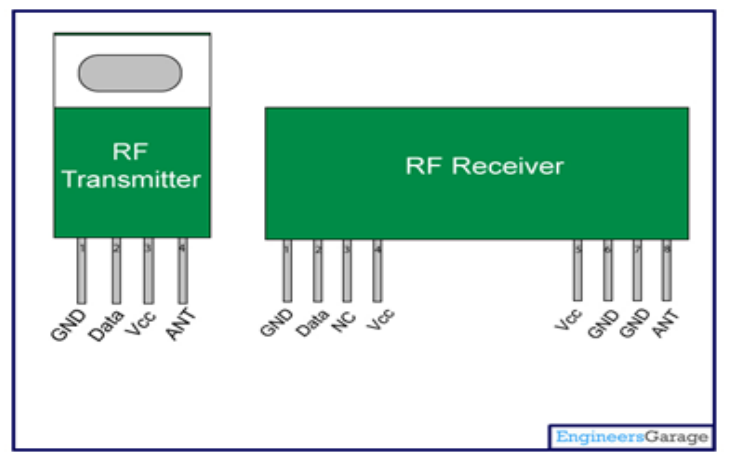

Figure 1: Pin Diagram for RF transmitter and receiver 
Pin Description:

\begin{tabular}{|c|c|c|}
\multicolumn{3}{|c}{ Table 1: RF Receiver } \\
\hline Pin no. & Function & Name \\
\hline 1 & Ground (0V) & Ground \\
\hline 2 & Serial data output pin & Data \\
\hline 3 & $\begin{array}{c}\text { Linear output pin; not } \\
\text { connected }\end{array}$ & NC \\
\hline 4 & Supply voltage; $5 \mathrm{~V}$ & Vcc \\
\hline 5 & Supply voltage; $5 \mathrm{~V}$ & Vcc \\
\hline 6 & Ground (0V) & Ground \\
\hline 7 & Ground (0V) & Ground \\
\hline 8 & Antenna input pin & ANT \\
\hline
\end{tabular}

Table 2. RF Transmitter

\begin{tabular}{|c|c|c|}
\hline Pin no. & Function & Name \\
\hline 1 & Ground (0V) & Ground \\
\hline 2 & Serial data output pin & Data \\
\hline 3 & Supply voltage; $5 \mathrm{~V}$ & Vcc \\
\hline 4 & Antenna output pin & ANT \\
\hline
\end{tabular}

The transmitter/receiver $(\mathrm{Tx} / \mathrm{Rx})$ pair operates at a frequency of $434 \mathrm{MHz}$.An RF (Radio Frequency) transmitter receives serial data and then transmits it wirelessly through RF through its antenna connected at pin4. The transmission takes place at the rate of $1 \mathrm{Kbps}-10 \mathrm{Kbps}$. The transmitted data is received by an RF receiver operating at the same frequency as that of the transmitter. The RF module is frequently used along with a pair of encoder/decoder. The encoder is used for encoding parallel data for transmission feed while reception is decoded by a decoder. There are some commonly used encoder/decoder pair ICs HT12E-HT12D, HT640-HT648, etc. The pin diagram for RF transmitter and receiver is shown in Figure 1 .

\section{LITERATURE SURVEY}

A security guard is a person who is paid to protect assets, property or people. Security guard is a civilian personnel employed usually privately and formally. Security guard are generally uniformed and act to protect property by maintaining a high visibility presence to deter illegal and inapt actions, observing either directly, through sentry, or by watching alarm systems or by video cameras for signs of crime, disorder or fire; then taking action and reporting any incidents to their client and emergency services as proper.

Until the $1980 \mathrm{~s}$, the term watchman was more commonly applied to provide security, a usage dating back to at least the middle age in Europe. The term watchman was carried over to North America where it was interchangeable with nightwatchman until both terms were replaced with the modern refuge-based title. Security guards are at times regarded as fulfilling a private policing function.
Closed-circuit television (CCTV) is the use of video cameras to transmit a signal to a particular place, on a partial set of monitors. It differs from broadcast television in that the signal is not openly transmitted, though it may employ point to point (P2P), point to multipoint, or mesh wireless link. Although approximately all video cameras fit the definition, the term is most often applied to those used for surveillance in areas that may need monitoring such as casinos, airports, banks, convenience stores and military installations. Video telephony is seldom called "CCTV" but the use of video in distance learning, where it is an imperative tool, is frequently so called.

In manufacturing plants, CCTV paraphernalia may be used to observe parts of a process from a central control room for example where the environment is not suitable for humans. CCTV systems may operate constantly or only as required to monitor a particular event. A more superior form of CCTV, utilizing digital video recorders (DVRs), provides recording for possibly many years, with a performance options and variety of quality and extra features such as email alerts and motion-detection. More freshly, decentralized IP-based CCTV cameras are equipped with megapixel sensors, support internal flash for completely stand-alone operation or recording directly to network-attached storage devices. Surveillance of the communal using CCTV is particularly common in many areas around the world including the United Kingdom, where there are seemingly more cameras per person than in any other country in the world. There and in another place, its increasing use has triggered a debate about security versus privacy.

\section{SYSTEM ARCHITECTURE}

The architecture is divided into two types:

1. Web Based Application

2. Windows Based Application

\subsection{Web Based Application}

The topic monitoring system for prisoner uses web based application to send SMS using internet to the officer if prisoner tries to escape or harm the bracelet.

\subsection{Windows Based Application}

The system even offers windows based application to receive signal that is send by the transmitter and also the application will receive the location information of bracelet through GPS tracker. So accordingly the officer will come to know about the prisoner's location through GPS if he/she tries to escape from jail.

\subsection{Working}

The working is explained with an example. Suppose considering a prisoner. The actor is prisoner; the bracelet will tied on prisoner wrist to monitor him. If the prisoner is inside the campus the signal i.e. frequency will be in some range limit for that bracelet. 


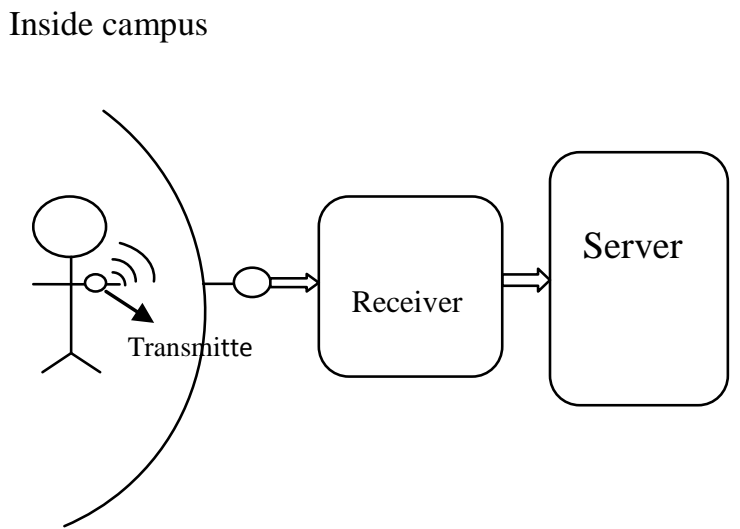

Figure 2: Architecture for Inside Campus

The transmitter will transmit the signal that will be received by the receiver. The transmitter will be implemented on the bracelet and the receiver will be implemented on the server. For inside campus there will be some rang limit given which will indicate that the prisoner will be in jail. And if the prisoner tries to harm the bracelet the sever will generate an alarm even the message will be send to the police officer of jail on his mobile so accordingly the prisoner will be monitored without any CCTV Camera.
Outside campus

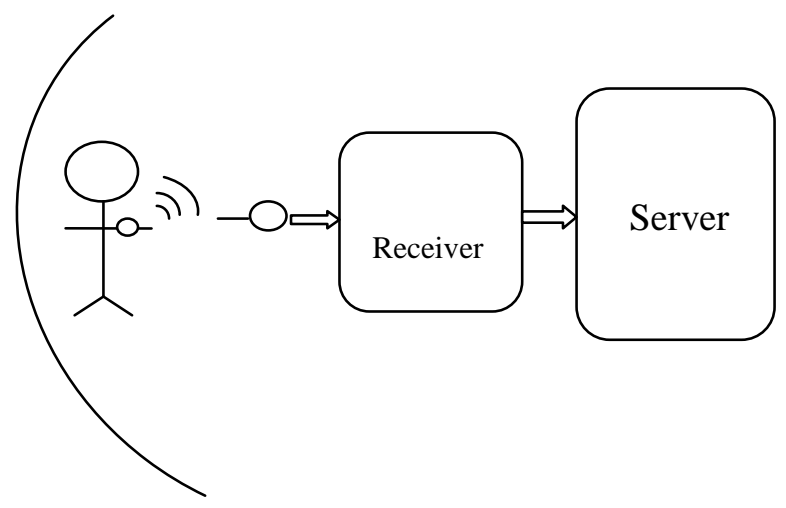

Figure 3: Architecture for outside Campus

Similarly, for outside campus if the prisoner tries to run out of jail i.e. tries to escape from jail. The frequency range will be break out and will generate the alarm and message will be sent to officer to indicate the prisoner location when he escapes. The GPS will be helpful to get the location of the prisoner if he/she tries to escape. In this case GPS plays an important role for outside campus monitoring.GPS (Global Positioning System): GPS is a space-based navigation system that provides location, time information, anywhere on or near the Earth. GPS system is made up of a Network of 24 satellites placed into orbit.

In the topic of monitoring system for prisoner, GPS module is used for tracking the prisoner when he/she tries to escape the campus. GPS system is activated when the prisoner goes outside the campus then using GPS module the jailer will get the position of the prisoner with the help of the longitude and latitude so that can easily find out the prisoner. In GPS module a CC250 is used for wireless transmission of signal. The CC250 which is used in system is in pair of transmitter and receiver. The transmitter of CC250 is implemented at GPS transmitter side and Receiver of CC250 is implemented at the receiver side of GPS module where, receiving signal using ARDUINO. ARDUINO is used for receiving signal from GPS module which is implemented at transmitter side.
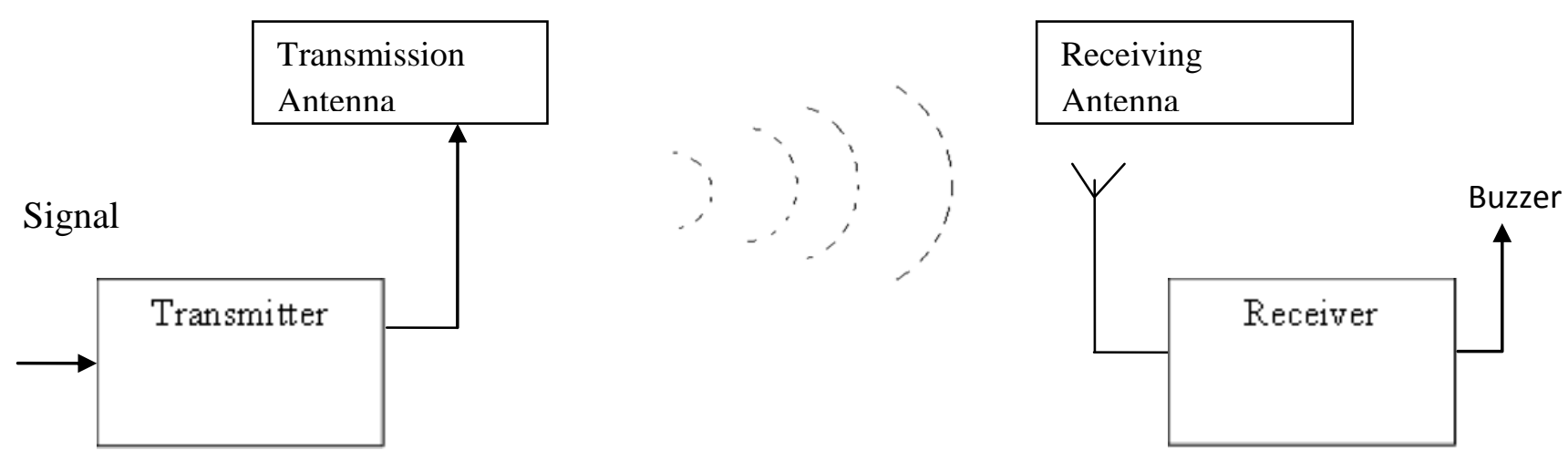

Figure 4: System Structure 


\section{COMPONENT}

\subsection{Transistor}

A voltage or current applied to one pair of transistor's terminal changes the current through another pair of terminal. Transistor is a Semiconductor device used to amplify and it is also used for switching electronic signals and electrical power.

\subsection{Resistor}

Resistor is a passive two-terminal electrical component that implements electrical resistance as a circuit element. The current through a resistor is direct proportion to voltage across the resistor's terminal.

\subsection{Relay}

Relay is used where it is necessary to control a circuit by a low-power signal or where several circuits must be controlled by one signal.

\subsection{PCB (Printed Circuit Board)}

Mechanically supports and electrically connects electronic components using conductive tracks.

\subsection{LED (Light Emitting Diode)}

LED is used as indicator lamps in many devices.

\subsection{LPT (Licensed Penetration Tester)}

LPT a parallel port is a type of interface found on computer for connecting peripherals.

\subsection{Diode}

Diode is a two-terminal electronic component with asymmetric conductance. It has low resistance to current flow in one direction and high resistance in other.

\subsection{Transmitter}

Transmitter is an electronic device which generates signals for communication purpose. It generates radio frequency alternating current which is then applied to antenna.

\subsection{Receiver}

Receiver is an electronic device that receives signals and converts that information into usable form. The information produced by receiver may be in the form of sound, data or images.

\subsection{Buzzer}

Buzzer is an audio signaling device.

Above components are used to design the system which is helpful to monitor that the prisoner is present in the jail or not. Buzzer is use to indicate that the prisoner is run away from jail so that immediate actions can be taken to catch the prisoner who tries to escape.

\section{CONCLUSION}

In today's world security factor plays an important role in every one's life .The topic which is being implementing is helpful for security purpose. At first CCTV camera where placed around the jail. But though the prisoner any how manages to try to escape from jail, as CCTV camera in jail where place on some fixed position.

The security bracelet is tied on prisoner's wrist, if he /she tries to escape from jail it will buzzer or send message to the jailer and the immediate actions can be then taken to catch the prisoner who tries to escape.GPS makes it easier to trace the prisoners latitude and longitude.

\section{ACKNOWLEDGMENTS}

This paper topic could never have completed without support and assistance of many people. First and foremost, would like to express deepest gratitude to the guide Prof. P. A. Kale for his excellent guidance, valuable solution and kind of encouragement in academics. And are very thankful to Prof. P. A. Kale and all staff of Late G.N.S.COE, Nasik.

\section{REFERENCES}

[1] Jinaporn ,N .Dept. of Electronics. \& Electron. Eng., Ubonratchathani Univ., UbonRatchathani , "Security system against asset theft by using Radio Frequency Identification Technology ",IEEE Aug.2009.

[2] Modares ,H. ; Dept. of Comput. Syst. \& Technol., Univ. of Malaya, Kuala Lumpur, Malaysia ;Salleh, R.; Moravejosharieh, A., "Overview of Security Issues in Wireless Sensor Networks”,IEEE Aug. 20-22 Sept. 2011.

[3] K. Domdouzis, B. Kumar and C. Anumba, "RadioFrequency Identification (RFID) applications: A brief introduction," Advanced Engineering Informatics, vol. 21 , issue 4, pp. 350-355, October 2007.

[4] M. Ward, R. van Kranenburg, "RFID: Frequency, standards, adoption and innovation", JISC Technology and Standards Watch, May 2006.

[5] R.Angeles, "RFID technologies: Supply-chain applications and implementation issues," Information Systems Management, vol.22, issue ,pp.51-65, 2005.

[6] W.M.G. Wismans,. "Identification and registration of animals in the European Union," Computers and Electronics in Agriculture, vol. 24, issue 1-2,pp.99108,1999

[7] G. Adams, "Pharmaceutical manufacturing: RFID reducing errors and effort," Filtration \& Separation, vol. 44, issue 6, pp17-19,July-August 07.

[8] M. Venkatesan, Z. Grauer, "Leveraging radio frequency identification (RFID) technology to improve laboratory information management," American Laboratory, vol.36, issue 18, pp.11-14, 2004.

[9] M. Lu, W. Chen, X. Shen, H.C. Lam and J. Liu, "Positioning and tracking construction vehicles in highly dense urban areas and building construction sites," Automation in Construction, vol. 16, issue 5, pp. 647656,August2007. 